\title{
STABILITY ANALYSIS OF A LOTKA-VOLTERRA TYPE PREDATOR-PREY SYSTEM INVOLVING ALLEE EFFECTS
}

\author{
HÜSEYIN MERDAN ${ }^{1}$
}

(Received 21 August, 2010; revised 2 March, 2011)

\begin{abstract}
We present a stability analysis of steady-state solutions of a continuous-time predatorprey population dynamics model subject to Allee effects on the prey population which occur at low population density. Numerical simulations show that the system subject to an Allee effect takes a much longer time to reach its stable steady-state solution. This result differs from that obtained for the discrete-time version of the same model.

2000 Mathematics subject classification: primary 34D20; secondary 92D25, 34A34. Keywords and phrases: Allee effects, population dynamics, stability analysis, predatorprey model.
\end{abstract}

\section{Introduction}

Time evolution of populations is generally modelled by either continuous-time models, or discrete-time models often obtained via a time discretization of the former using the Euler method. Although a discrete-time population model presents a richer dynamical picture (specifically, in terms of numerical simulation), continuous-time population models are more appropriate to nature except for nonoverlapping generations. While, in general, discrete-time models closely approximate continuous-time models, they result in different stability behaviour under Allee effects [4, 10, 15].

The Allee effect, first introduced by Allee [1] in 1931, represents a negative density dependence where the population growth rate is reduced at low population size. It may be caused by a number of factors including difficulties in finding mates, social dysfunction at small population sizes, inbreeding depression and food exploitation. Such effects may be observed in different organisms, including vertebrates, invertebrates and plants. The effect usually saturates or vanishes as the population size increases.

Allee effects have recently been studied intensively in population dynamics. Previous studies have demonstrated that Allee effects can have important dynamical

\footnotetext{
${ }^{1}$ TOBB University of Economics and Technology, Faculty of Arts and Sciences, Department of Mathematics, Söğütözü 06530, Ankara, Turkey; e-mail: merdan@etu.edu.tr.

(c) Australian Mathematical Society 2011, Serial-fee code 1446-1811/2011 \$16.00
} 
consequences on local stability analysis of population models. They may have either a destabilizing or a stabilizing role: the local stability of an equilibrium solution may be changed from stable to unstable or vice versa [2-5, 7-15]. It is also possible that for a population subject to an Allee effect, the system may take a much longer time to reach its steady-state solution even when it is a stable equilibrium solution $[4,15]$.

In this paper, we investigate the impact of Allee effects on the stability of equilibrium solutions of the following continuous-time predator-prey model, a LotkaVolterra type predator-prey system:

$$
\frac{d x}{d t}=r x(1-x)-a x y, \quad \frac{d y}{d t}=a y(x-y),
$$

where $x(t)$ and $y(t)$ denote the densities of prey and predator populations at time $t$, respectively, and $r, a$ are positive parameters. The term $r x(1-x)$ represents the growth rate of the prey population without predators in the environment. The prey population decreases due to predation denoted by the term $a x y$, in which $a$ is the conversion efficiency of prey into predators and $a x$ is the predator functional response, which represents the number of prey individuals consumed per unit area per unit time by an individual predator. From a biological point of view, the term $a y(x-y)$ in the second equation above represents predator density variation with respect to the prey population density. In the absence of predators (that is, when $y=0),(1.1)$ reduces to the well-known continuous-time logistic equation.

The aim of this paper is to present a stability analysis of equilibrium solutions of this continuous-time model with and without Allee effects. The stability conditions are compared through analysis supported by numerical simulations. The impact of Allee effects on the stability of steady-state solutions of the continuous-time model above is compared to that obtained for the discrete-time model of this predator-prey system studied by Çelik and Duman [2], which showed that Allee effects increase the stability of the fixed points.

The paper is organised as follows. Section 2 first gives a characterization of the stability of the equilibrium solutions of (1.1) and investigates their stability behaviour. Following this, a stability analysis of the equilibrium points of the same model subject to an Allee effect on the prey density is presented. Section 3 presents some numerical simulations concerning the stability of our models.

\section{Existence and stability of fixed points}

This section concerns the stability analysis of steady-state solutions of the population model (1.1) with and without Allee effects. Defining

$$
f(x, y):=r x(1-x)-a x y \quad \text { and } \quad g(x, y):=a y(x-y),
$$

the steady-state solutions of (1.1) (without Allee effects) are obtained by solving the equations $f(x, y)=0$ and $g(x, y)=0$. The model has three steady-state solutions: $(0,0),(1,0)$ and $\left(x^{*}, y^{*}\right):=(r /(a+r), r /(a+r))$. The local stability of these solutions 
is discussed below. However, from a biological point of view, we are mainly interested in the positive equilibrium solution.

The variational matrix of the continuous-time system (1.1) at an equilibrium solution $(x, y)$ is

$$
J(x, y)=\left(\begin{array}{ll}
f_{x}(x, y) & f_{y}(x, y) \\
g_{x}(x, y) & g_{y}(x, y)
\end{array}\right)=\left(\begin{array}{cc}
r-2 r x-a y & -a x \\
a y & a x-2 a y
\end{array}\right) .
$$

The characteristic equation of the variational matrix is

$$
\lambda^{2}-\operatorname{tr}(J) \lambda+\operatorname{det}(J)=0 .
$$

For a continuous-time system, asymptotic stability of an equilibrium solution is satisfied as long as $\operatorname{tr}(J)<0$ and $\operatorname{det}(J)>0$, so that both eigenvalues have negative real parts $[6,12]$. For the steady-state solution $(0,0), \operatorname{tr}(J(0,0))=r$, which is positive since we assume that $r>0$, and $\operatorname{det}(J(0,0))=0$, so that the corresponding eigenvalues are $\lambda=0$ and $\lambda=r$. Similarly, $\operatorname{tr}(J(1,0))=-r+a$ and $\operatorname{det}(J(1,0))=-r a$ for the steadystate solution $(1,0)$, so the eigenvalues are $\lambda=2 r>0$ and $\lambda=-2 a$. Therefore, both steady-state solutions are locally unstable. In addition, the equilibrium solution $(0,0)$ is nonhyperbolic.

For the positive steady-state solution $\left(x^{*}, y^{*}\right)=(r /(a+r), r /(a+r))$, one calculates $\operatorname{tr}\left(J\left(x^{*}, y^{*}\right)\right)=-r$ and $\operatorname{det}\left(J\left(x^{*}, y^{*}\right)\right)=a r^{2} /(r+a)$. Since $a$ and $r$ are positive, $\operatorname{tr}(J)<0$ and $\operatorname{det}(J)>0$, and hence this steady-state solution is locally asymptotically stable.

We now analyse the stability of steady-state solutions of the system subject to an Allee effect on the prey population. The model involving the Allee effect is

$$
\frac{d x}{d t}=r \alpha(x) x(1-x)-a x y, \quad \frac{d y}{d t}=a y(x-y),
$$

where $\alpha(x):=x /(\beta+x)$ is the term for the Allee effect and $\beta>0$ can be called the "Allee effect constant". The larger $\beta$ is, the stronger the Allee effect will be, and the slower the per capita growth rate of the prey population: by introducing the Allee effect into the model (1.1), the per capita growth rate of the prey population is reduced from $r x(1-x)$ to $r(x /(\beta+x)) x(1-x)$. Note that biological facts lead us to the following assumptions about the function $\alpha$ :

(1) if $x=0$ then $\alpha(x)=0$, that is, there is no reproduction without partners;

(2) $\alpha^{\prime}(x)>0$ for $x \in(0, \infty)$, that is, the Allee effect decreases as density increases;

(3) $\lim _{x \rightarrow \infty} \alpha(x)=1$, that is, the Allee effect vanishes at high densities.

Setting the right-hand sides in (2.1) equal to zero yields three equilibrium solutions: $(0,0),(1,0)$ and $\left(x_{*}, y_{*}\right):=((r-a \beta) /(a+r),(r-a \beta) /(a+r))$. Once again we are interested in the positive steady-state solution. Under the condition

$$
r-a \beta>0
$$

$\left(x_{*}, y_{*}\right)$ is a positive equilibrium solution; its local stability is discussed below. 
The Jacobian matrix of the system (2.1) at an equilibrium solution $(x, y)$ is

$$
J_{\beta}(x, y)=\left(\begin{array}{cc}
\frac{2 r x \beta+r x^{2}-3 r \beta x^{2}-2 r x^{3}-a y \beta^{2}-2 a y x \beta-a y x^{2}}{(\beta+x)^{2}} & -a x \\
a y & x a-2 a y
\end{array}\right) .
$$

The characteristic equation of the variational matrix $J_{\beta}(x, y)$ is again

$$
\lambda^{2}-\operatorname{tr}\left(J_{\beta}\right) \lambda+\operatorname{det}\left(J_{\beta}\right)=0 .
$$

Since $\operatorname{tr}\left(J_{\beta}(0,0)\right)=0$ and $\operatorname{det}\left(J_{\beta}(0,0)\right)=0$ for the equilibrium solution $(0,0)$, the corresponding eigenvalues are $\lambda_{1}=\lambda_{2}=0$. Thus $(0,0)$ is again nonhyperbolic. Similarly, $\operatorname{tr}\left(J_{\beta}(1,0)\right)=(a+a \beta-r) /(\beta+1)$ and $\operatorname{det}\left(J_{\beta}(1,0)\right)=-r a /(\beta+1)$ for the equilibrium solution $(1,0)$, so that $\operatorname{det}\left(J_{\beta}(1,0)\right)<0$ (because $a, r, \beta>0$ ). Therefore, both steady-state solutions $(0,0)$ and $(1,0)$ are again locally unstable.

Let us now analyse the stability of the positive steady-state solution $\left(x_{*}, y_{*}\right)$. The Jacobian matrix (2.3) becomes

$$
J_{\beta}\left(x_{*}, y_{*}\right)=\left(\begin{array}{cc}
\frac{(r-a \beta)\left(2 r a \beta+a^{2} \beta-r^{2}\right)}{r(r+a)(\beta+1)} & -\frac{a(r-a \beta)}{r+a} \\
\frac{a(r-a \beta)}{r+a} & -\frac{a(r-a \beta)}{r+a}
\end{array}\right) .
$$

One calculates that

$$
\operatorname{tr}\left(J_{\beta}\left(x_{*}, y_{*}\right)\right)=-\frac{(r-a \beta)^{2}}{r(\beta+1)} \quad \text { and } \quad \operatorname{det}\left(J_{\beta}\left(x_{*}, y_{*}\right)\right)=\frac{(r-a \beta)^{3} a}{r(r+a)(\beta+1)} .
$$

Hence $\operatorname{tr}\left(J_{\beta}\right)<0$ and $\operatorname{det}\left(J_{\beta}\right)>0$ at this solution under the condition (2.2). Thus, the positive steady-state solution $\left(x_{*}, y_{*}\right)=((r-a \beta) /(a+r),(r-a \beta) /(a+r))$ is locally asymptotically stable as long as condition (2.2) is satisfied.

Remark. From the stability analysis of the steady-state solutions of (1.1) and (2.1), we observe the following.

(1) If $\beta=0$ in $\alpha(x):=x /(\beta+x)$, then (2.1) reduces to (1.1).

(2) If the positive parameters $r, a$ and $\beta$ satisfy $\beta \geq(r / a)$, then (2.1) has no positive equilibrium solution. However, (1.1) has a positive steady-state solution which is locally asymptotically stable. This is one of the impacts of Allee effects.

(3) If the positive parameters $r, a$ and $\beta$ satisfy $\beta<(r / a)$, then (2.1) has a positive equilibrium solution which is locally asymptotically stable. However, the numerical simulations in the next section show that the system subject to an Allee effect takes a longer time to reach its steady-state solution than the system (1.1). Moreover, the numerical simulations once again show that the Allee effect reduces the population densities of both predator and prey at the steady state even if it seems from the analytical results that Allee effects have no destabilizing role (see Figures 1 and 2).

(4) In contrast to the results concluded for the continuous-time model, Allee effects have a stabilizing role in the discrete-time version of (1.1) [2]. 

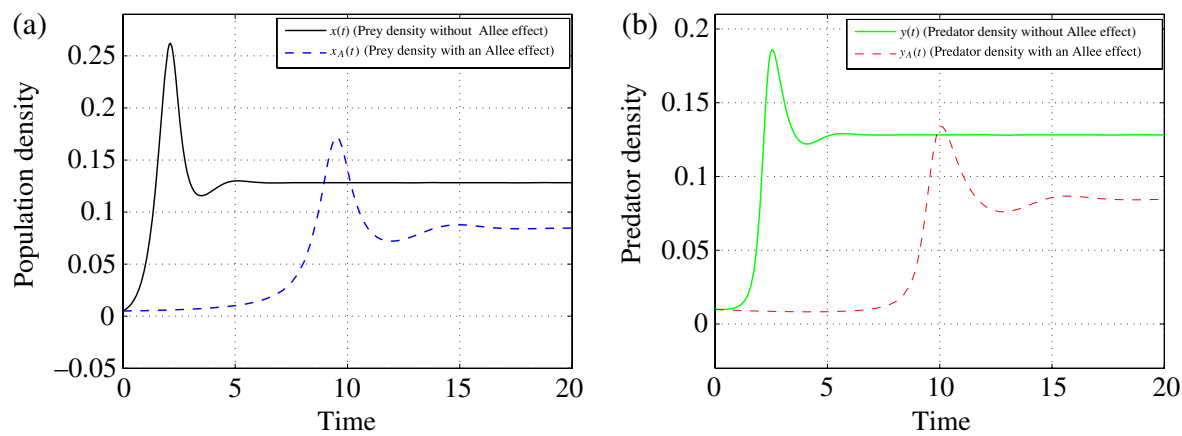

Figure 1. (a) Time-population density graphs of prey densities without the Allee effect, denoted by $x(t)$ (solid line), and with the Allee effect, denoted by $x_{A}(t)$ (dashed line). (b) Time-population density graphs of predator densities without the Allee effect, denoted by $y(t)$ (solid line), and with the Allee effect, denoted by $y_{A}(t)$ (dashed line). The initial conditions in both graphs are $x(0)=0.005$ and $y(0)=0.01$, and parameters are $r=2.5, a=17$ and $\beta=0.05$.

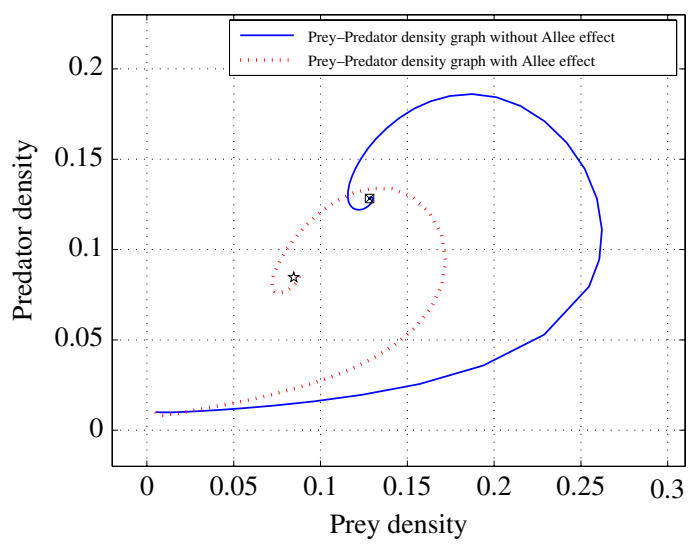

FigURE 2. Population density graphs of prey versus predator species with and without an Allee effect. The initial conditions in both graphs are $x(0)=0.005$ and $y(0)=0.01$. Parameters are $r=2.5, a=17$ and $\beta=0.05$.

\section{Numerical simulations}

We now report on numerical simulations of the competitive population model with and without Allee effects, defined by (2.1) and (1.1), respectively. We used the MATLAB ODE package for these computations.

In each of the simulations below, parameters are set as $r=2.5, a=17$ and $\beta=0.05$, which are the growth rate and the conversion efficiency of prey into predators, and the Allee effect constant, respectively. The initial conditions are taken as $x(0)=0.005$ and $y(0)=0.01$, which are initial prey and predator densities, respectively. The steady-state solutions of the systems corresponding to these parameter values are $x^{*}=y^{*} \cong 0.1282$ for (1.1) and $x_{*}=y_{*} \cong 0.0846$ for (2.1). 

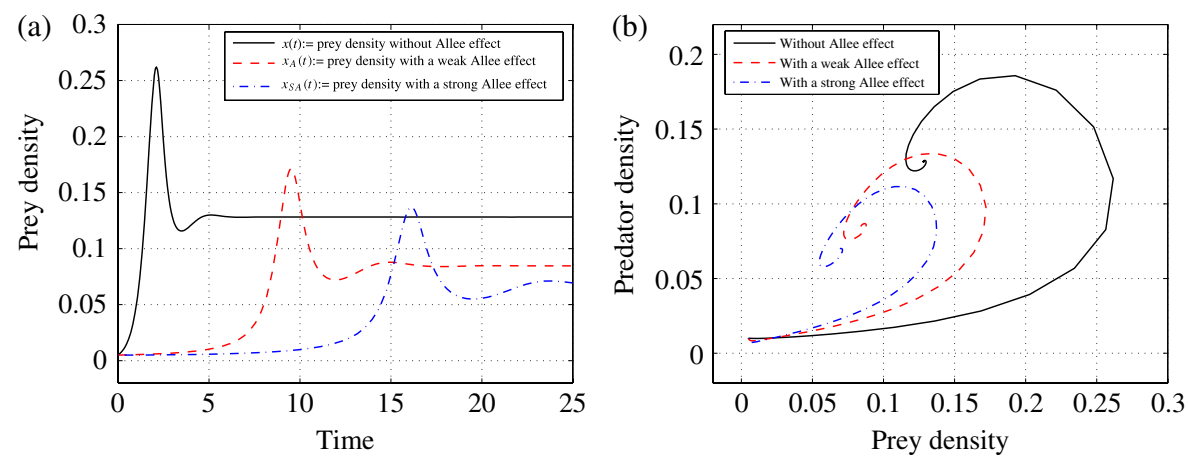

Figure 3. (a) Time-population density graphs of the prey population with and without Allee effects, denoted by $x_{A}$ and $x_{S A}$. (b) Population density graphs of prey density versus predator density with and without Allee effects. The initial conditions in both graphs are $x(0)=0.005$ and $y(0)=0.01$. Parameters are $r=2.5, a=17, \beta_{A}=0.05$ and $\beta_{S A}=0.07$.

Figure 1 shows the impact of Allee effects on the local stability of the positive equilibrium solution of the population model defined by (1.1). In Figure 1(a), we compare the time evolution of the prey density obtained from (1.1) with that of the prey density obtained from (2.1). Figure 1(b) shows a similar comparison for the predator densities with and without the Allee effect $\alpha(t):=x(t) /(\beta+x(t))$. One can see that the Allee effect reduces the population densities of both species at the stable steady-state solutions. Also, both graphs show that the trajectories of the system subject to an Allee effect (dashed lines) approach the equilibrium solution more slowly than in the model without the Allee effect (solid line).

The result obtained is different from that obtained for the discrete-time version of the same model by Çelik and Duman [2], who have illustrated that Allee effects increase the local stability of equilibrium points of the discrete-time predator-prey model while the densities of both species at the equilibrium point decrease.

Figure 2 shows the trajectories of the prey density, $x(t)$, versus the predator density, $y(t)$, around the positive equilibrium solutions. Figure 3 verifies that the larger $\beta$ is, the stronger the Allee effect will be. In this simulation, we take the Allee effect constants as $\beta=0.05$ for the weak Allee effect and $\beta=0.07$ for the strong Allee effect. We see that the system takes a much longer time to reach its stable steady-state solution as $\beta$ increases in the Allee effect $\alpha(t):=x(t) /(\beta+x(t))$.

\section{Conclusions and discussion}

Previous studies have demonstrated that Allee effects play an important role in the stability analysis of equilibrium points or steady-state solutions of a population dynamics model [2-5, 7-11, 13-15]. An Allee effect may have a stabilizing or a destabilizing effect on population dynamics: the equilibrium points of a system could be changed from unstable to stable or vice versa. The system subject to an Allee effect, even when it is stable, may take a much longer time to reach its stable state. 
This paper has focused on the stability analysis of a second-order continuoustime predator-prey model subject to an Allee effect on the prey population. We first characterized the stability of equilibrium solutions of the population model defined by (1.1). Then, imposing an Allee effect on the system in (2.1), we also studied the stability of the new steady-state solutions. Numerical simulations show that when one introduces an Allee effect into the system, the system takes a longer time to reach its stable steady-state solution, and the equilibrium densities of both species at the stable steady state are reduced.

\section{References}

[1] W. C. Allee, Animal aggregations: a study in general sociology (University of Chicago Press, Chicago, 1931).

[2] C. Çelik and O. Duman, "Allee effect in a discrete-time predator-prey system", Chaos, Solitons Fractals 90 (2009) 1952-1956, doi:10.1016/j.chaos.2007.09.077.

[3] C. Çelik, H. Merdan, O. Duman and Ö. Akın, "Allee effects on population dynamics with delay", Chaos, Solitons Fractals 37 (2008) 65-74, doi:10.1016/j.chaos.2006.08.019.

[4] O. Duman and H. Merdan, "Stability analysis of continuous population model involving predation and Allee effect", Chaos, Solitons Fractals 41 (2009) 1218-1222, doi:10.1016/j.chaos.2008.05.008.

[5] M. S. Fowler and G. D. Ruxton, "Population dynamic consequences of Allee effects", J. Theoret. Biol. 215 (2002) 39-46, doi:10.1006/jtbi.2001.2486.

[6] J. Hale and H. Koçak, Dynamics and bifurcation (Springer, Berlin, 1991).

[7] S. R.-J. Jang, "Allee effects in a discrete-time host-parasitoid model with stage structure in the host", Discrete Contin. Dyn. Syst. B 8 (2007) 145-159, doi:10.3934/dcdsb.2007.8.145.

[8] R. López-Ruiz and D. Fournier-Prunaret, "Indirect Allee effect, bistability and chaotic oscillations in a predator-prey discrete model of logistic type", Chaos, Solitons Fractals 24 (2005) 85-101, doi:10.1016/j.chaos.2004.07.018.

[9] M. A. McCarthy, "The Allee effect, finding mates and theoretical models", Ecol. Model. 103 (1997) 99-102, doi:10.1016/S0304-3800(97)00104-X.

[10] H. Merdan and O. Duman, "On the stability analysis of a general discrete-time population model involving predation and Allee effects", Chaos, Solitons Fractals 40 (2009) 1169-1175, doi:10.1016/j.chaos.2007.08.081.

[11] H. Merdan, O. Duman, Ö. Akın and C. Çelik, "Allee effects on population dynamics in continous (overlaping) case", Chaos, Solitons Fractals 39 (2009) 1994-2001, doi:10.1016/j.chaos.2007.06.062.

[12] J. D. Murray, Mathematical biology (Springer, New York, 1993).

[13] I. Scheuring, "Allee effect increases the dynamical stability of populations", J. Theoret. Biol. 199 (1999) 407-414, doi:10.1006/jtbi.1999.0966.

[14] P. A. Stephens and W. J. Sutherland, "Consequences of the Allee effect for behaviour, ecology and conservation", Trends Ecol. Evol. 14 (1999) 401-405, doi:10.1016/S0169-5347(99)01684-5.

[15] S. R. Zhou, Y. F. Liu and G. Wang, "The stability of predator-prey systems subject to the Allee effects", Theor. Popul. Biol. 67 (2005) 23-31, doi:10.1016/j.tpb.2004.06.007. 